\title{
Privacy and Interruptions in Team Awareness Systems
}

\author{
Carsten Röcker and Carsten Magerkurth \\ Fraunhofer IPSI, AMBIENTE - Smart Environments of the Future \\ Dolivostraße 15, D-64293 Darmstadt, Germany \\ \{roecker, magerkurth\} @ipsi.fraunhofer.de
}

\begin{abstract}
Several evaluations of team awareness systems showed, that interruptions and privacy violations during usage often lead to the rejection of the system by users. Most authors argue that this rejection is due to a fundamental dual trade-off between sending awareness information and privacy, and between receiving awareness information and disruption or resource consumption. While the assumption of a fundamental trade-off is widely accepted in state-of-the-art research, this paper disputes the predominant hypothesis. Instead, it is argued, that the trade-off is not of fundamental nature, but caused by neglecting elementary aspects in the design process. In order to verify this line of argument, a novel interface concept for mediating socioemotional awareness information is presented. To verify the validity of the conceptual approach, several evaluations were conducted. The evaluations verified the approach of this paper and showed, that a cautious interface design can enhance user privacy in multi-user awareness systems and minimize disruptive effects on primary tasks, without reducing awareness mediation and usability.
\end{abstract}

Keywords: Privacy, Interruptions, Team Awareness Systems, Dual Trade-Off, Evaluation.

\section{Introduction and Motivation}

In intellectual teamwork, implicit communication in form of mutual awareness is an important requirement for a shared understanding and knowledge about ongoing and past activities within a team [12]. Mutual awareness usually leads to informal interactions, spontaneous connections, and the development of shared cultures, all important aspects of maintaining working relationships [1]. Especially information about presence and availability of remote colleagues is of high value during the daily work process. In a local work environment, this information is continuously available and picked up by those present. Teams, which are geographically distributed, by their nature, are denied the informal information gathered from a physical shared workspace [3]. These shortcomings led to the development of a variety of so-called 'awareness systems', dedicated applications for supporting awareness between different groups and places. A number of these systems have been tested in real world situations [e.g., 8, 1, 13]. Although it was shown that the installations had some success in getting people to communicate more easily, all systems were abandoned 
after the demonstration period [7]. The rejection of the systems was due to serious usability problems caused by recurring interruptions and privacy violations.

In the literature, this problem is often referred to as the dual trade-off between the level of awareness and the potential for privacy intrusion and disruption of one's current tasks. The first trade-off of 'Informativeness vs. Privacy' is caused by the fact, that, if the current status of a person is conveyed fully enough to be useful to others, it often violates that person's privacy [5]. The second trade-off describes the problem of 'Information vs. Interruption'. In general, the more information one receives about the activities of remote colleagues, the more awareness is mediated, but the greater the chances that the transmitted information will become a disturbance to the primary task [2]. Like most other authors, Hudson and Smith [2] argue, that this dual trade-off between sending awareness information and privacy, and between receiving awareness information and disruption or resource consumption, is fundamental at some level. While the assumption of a fundamental trade-off is widely accepted in state-of-the-art research, this paper disputes the predominant hypothesis. Instead, it is argued, that the trade-off is not of fundamental nature, but caused by neglecting two elementary aspects in the design process.

First, current implementations do not take into account, that awareness information is perceived as a continuous secondary task. While content-oriented communication, like e-mail or chat, is usually performed as a primary task, the perception of environmental information is done as a secondary task. Most team awareness systems do not consider this fact and try to mediate awareness as a primary task, requiring full attention or considerable input from the user. Second, the increased local mobility of the team members requires the information to be displayed in public and semi-public areas. With the transition from an individual to a group situation, new privacy problems arise, which are not adequately approached in the design of current awareness systems. Using traditional single-user interface policies to provide personalized information in public spaces, will inevitably lead to privacy violations.

\section{Goal and Concept}

The goal of this paper is to show, that the problems described above are not inherent in the information itself or its processing, but caused by the way the data are collected and represented. In order to verify this hypothesis, a novel interface concept for mediating socio-emotional awareness information in group situations was developed.

The conceptual design process was guided by two goals. First, it was aimed to provide users with 'lightweight' awareness devices, that help members of a distributed team to communicate in a natural way. In contrast to most existing approaches, awareness should be provided via a natural communication channel, that enables people to be aware of each other, in a subtle, warm and expressiveness way, which can be easily perceived on a human level. Second, the interfaces should be adapted to the changing requirements of emerging office concepts as well as to the increased mobility of employees within the work environment. As office workers get more and more mobile within the office space, the conceptual system design aims to support awareness and informal communication through natural interaction in public areas, using intuitive interfaces integrated into an open office landscape. 
This is achieved by combining various artefacts, which are integrated into a smart office environment and tailored to the needs of distributed teams. Ambient displays and sensors are embedded into the physical surrounding to communicate information and support implicit interaction mechanisms. These stationary artefacts are complemented by personal mobile devices, that help users to preserve their privacy in public space and access personalized information.

\section{Novel Interfaces for Mediating Awareness Information}

Based on the conceptual approach, different prototypes of mobile and stationary artefacts were developed, which use a common sensing infrastructure to support user interaction. The following paragraphs give a brief overview over the different artefacts and their main functionalities. For details on the developed artefacts see [10] or [11]. To enable user-controlled identification processes as well as personal role management, a mobile control device called Personal.Aura was developed. The Personal.Aura is a mobile device enabling users to control their appearance in a smart environment by deciding on their own, whether they want to be 'visible' for remote colleagues, and if so, in which 'social role' they want to appear. The Personal.Aura is a compound artefact consisting of a Reader Module and several complementary ID Sticks (see Fig. 1). Every ID Stick symbolizes a different social role and contains a unique identification code. If people want to signal their presence to remote team members, they can do so by simply connecting a specific ID Stick to the Reader Module. As soon as both parts are physically connected, the user is identified with the digital profile linked to the specific ID Stick. Disconnecting both parts immediately stops the identification process.

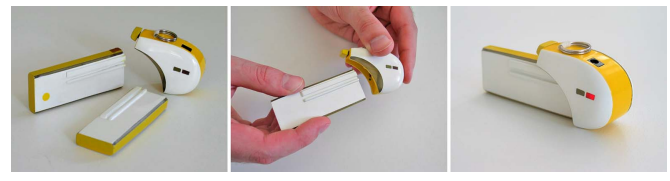

Fig. 1. Activation of the Personal.Aura artefact by connecting an ID Stick to the Reader Module

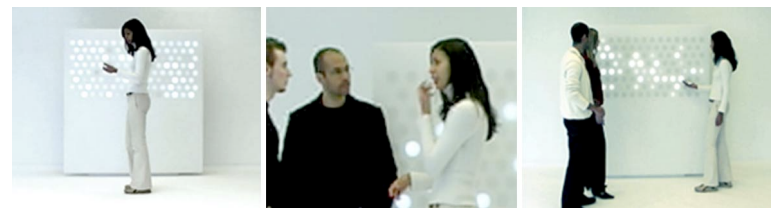

Fig. 2. Hello.Wall artefact showing different light patterns depending on the social situation

In order to represent the information in the users' environment, a wall-sized ambient display called Hello.Wall was developed. The Hello.Wall uses special light patterns to communicate information in an ambient and unobtrusive way. Sensors 
embedded in the Hello.Wall artefact enable context-dependent information representation. By restricting the reading range to a defined area around the artefact, it is ensured, that people are only sensed, when identification information is necessary in order to provide personalized services. The distance of an individual to the Hello.Wall determines the type of information visualized and forms of interaction, which are possible.

To demonstrate the potential of this approach, an exemplary pattern language (see Fig. 3) was developed to visualize information in an ambient and unobtrusive way. The goal was to improve workplace awareness and support opportunities for brief encounters between remote colleagues. In order to support awareness and informal communication, light patterns for the following information were designed: (1) the general mood of the remote team, (2) the general activity in the remote work space, (3) the presence and availability of certain team members, and (4) the interest for communication with a remote team member.
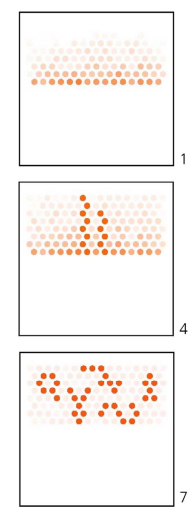
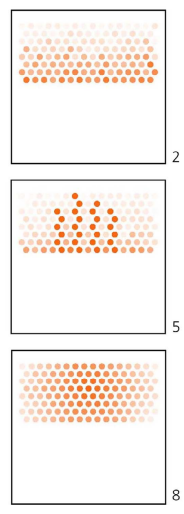

Fig. 3. Light patterns for the Hello.Wall
AMBIENT PATTERNS

Pattern 1 - 3

General Mood of the Remote Team

(bad, average, good)

Pattern 4 - 6

General Activity in the Remote

Workspace

(low, medium, high)

NOTIFICATION PATTERNS

Pattern 7

Presence and Availability of Certain Team Members

(personal signs of 5 team members)

Pattern 8+9

Interest for Communication with a Remote Team Member

(remote and local pattern)

The functionality of the Hello.Wall is complemented by mobile device named View.Port. The View.Port complements the functionality of the Hello. Wall artefact by providing additional in-depth information depending on the individual context. Through the private nature of its display, the View.Port enables users to access personal information in public spaces, without violating individual information privacy.

\section{Evaluations}

To verify the validity of the conceptual approach, and to confirm the added value of the technical prototypes compared to related research results, the developed artefacts were evaluated in a two-step process. To capture subjective, as well as performance related aspects, a combination of qualitative and quantitative evaluation techniques was employed. In a first experimental evaluation, the pattern representation used to visualize information at the Hello.Wall was compared to a video representation, which is currently the most-widely used representation form in multi-user awareness 
systems. Both representation methods were compared regarding their suitability to provide awareness information, their disruptive effects on work, as well as privacy concerns that arise during usage. In a second step, all artefacts were tested under realworld conditions for several weeks, in order to investigate their potential for supporting awareness and informal communication in a distributed team.

\subsection{Experimental Evaluation of the Representation Method}

Awareness information is usually delivered as a continuous secondary task, requiring users to rapidly and frequently switch between some other primary task and the awareness task. Consequently, the information should be presented in a subtle and non-distracting way. With the design of the Hello.Wall artefact, a novel approach regarding the visualization of awareness information was taken. Instead of using a traditional graphical display, the awareness information is visualized in the users' environment using ambient light patterns.

In contrast to the abstract patterns used to visualize information on the Hello. Wall, most multi-user systems show concrete visual information to support awareness. As video-based systems have proved to provide valuable information for distributed work, the light patterns developed for the Hello.Wall were compared to a simulated video connection. Following the current trend of providing peripheral awareness information in public and semi-public areas, both representation forms were visualized on a large public display. In a controlled experiment, users were confronted with both representation methods, using the same information and representation device. The goal of this comparison was to show, that the approach described in this paper is superior over traditional forms of information representation. To proof this, both forms of information representation were compared regarding their suitability to provide awareness information, their disruptive effects on work, as well as privacy concerns that arise.

In a simulated work situation, presence information about a fictive remote team was shown to the participants, while they were working on a primary task. A large display was used to show a recorded sequence, using the two different representation forms. Once the awareness information was presented in form of a video link, the other time the same information was visualized using Hello. Wall patterns.

In the video condition, the participants were shown a pre-recorded video sequence showing a fictive office with five employees. In the pattern condition, the presence of the team members was symbolized by different personal signs, that were displayed for the time the person is inside the office. To measure the effects of both representation forms regarding distraction and interruption, a special application was developed. The program consists of a computer game and an interface to indicate the perceived changes in the presence state. The computer game was designed to be particular sensitive to interruptions and distractions. Similar to a pinball machine, the player has to avoid balls from falling down by returning them with a paddle. With a simple mouse click the game is paused and the program switches to the 'awareness interface'. Here, the participants can indicate the presence or absence of the team members by clicking on their picture or personal sign. A second mouse click resumes the game. 
The performance in playing the game and in perceiving the presence information was analyzed through log files that were recorded during the whole evaluation. A purpose-build analyzing software continuously tracked the state of every button and automatically generated a graphical overview of the button states. The software also tracked the number of lost balls and periods during which the game was paused to determine the task performance. In addition, a video analysis was performed, to find out how often and how long the participants had to look at the display to pick-up the presence information. The duration and frequency of glances to the display were used as indicators for the degree of interruption. Besides these objective, performanceoriented criteria, it was also aimed to acquire subjective user impressions as more intuitive measures of mental workload. Therefore, different kinds of questionnaires were used to examine how the participants judged their own performance and how exhausting they found playing the game. If the method of presenting the awareness information influences the atmosphere and the concentration, there are probably differences in those subjective judgments depending on the representation technique.

During a two-step experiment both sequences were shown to $\mathrm{N}=47$ participants. The participants were divided into two groups, which differed only in the chronological order of the presented representation sequences. While the first group started with the pattern representation and saw the video representation in the second step, the order was the other way round for the second group. To make sure that the participants would be able to keep concentrated at a constant level, the test consisted of two parts of 23 minutes each. During this time the participants were asked to play the game and to keep track of the presence of each of the five people in the fictive remote office. Perceived changes concerning the presence of each remote team member had to be adjusted in the awareness interface immediately. After each test section, the participants filled out questionnaires, rating the recent representation concerning distraction and usefulness as well as their individual performance in playing the game and being aware of the remote colleagues. In the end, a third questionnaire was used to compare both representation forms. A detailed description of the evaluation procedure and results can be found in [4].

The evaluation showed, that the pattern representation used for the Hello.Wall significantly reduces distractions. The pattern representation was rated significantly more often as less distracting than the video representation. In addition, the performance in the game was less affected through the pattern representation. When using the video representation, the participants dropped highly significant more balls while receiving the awareness information, which has to be regarded as an indicator for a higher degree of distraction.

Using video for awareness mediation has the advantage, that users do not need any practice to understand the way of information representation. The log files proved, that the participants made fewer mistakes while interpreting the information when using the video representation. This might be explained by the fact, that the participants are used to remember the appearance of other humans, while they are not used to remember abstract patterns. Therefore, the participants had to cope with an additional load of learning and remembering the patterns while using the pattern representation. This additional load would be reduced, if the users already knew the patterns and their meaning. As an evaluation of the employed pattern language [6] showed a learning effect over time, it is likely, that the performance of interpreting 
the awareness information will improve with usage. For both representation forms, there was no significant difference in the frequency of temporal misinterpretation, where persons were perceived as 'present' although they were 'absent'. However, there were highly significant more temporal misinterpretations of 'absent' people while using the video representation.

In contrast, the recognition of persons leaving the fictive office was better, when using the video representation. But regarding the collaboration of teams, being aware of a person entering the office, and thus being available for immediate personal contact, is usually more valuable than recognizing, that someone had just left the office. One may wait for a colleague to enter the office to talk to him or to schedule something. When a team member leaves the office, information about the reason and duration of his absence are usually more helpful than just knowing, that he has gone. So, in this specific application domain, it might even be seen as an advantage, that only the more valuable presence information is perceived. This is also reinforced by the fact, that in the final questionnaire significant more participants rated the video representation as more distracting than the pattern representation. In addition, the privacy concerns, when using the pattern representation, are significantly lower. The evaluation of the questionnaire data showed further, that highly significant more participants preferred the pattern representation over the video representation, when making information about their own presence available to remote colleagues.

Hence, the evaluation supported the approach of using ambient patterns to visualize awareness information. It could be shown, that using a pattern representation significantly reduces distractions and privacy concerns, without negatively effecting the perception of awareness information.

\subsection{Living-Lab Evaluation of Artefacts}

In order to investigate the potential of the developed devices for supporting awareness and informal communication, all artefacts were tested in a living-lab evaluation over several weeks. The goal of the evaluation was, to create personal connections between remote team colleagues by establishing awareness moments, and supporting community interactions between both sides.

To evaluate the artefacts under real-world conditions, a symmetrical configuration of two Hello. Wall artefacts with additional video-conferencing facilities was installed at two remote work spaces of a distributed team [9]. The first set of artefacts was installed at Fraunhofer IPSI in Darmstadt (Germany), the second at the Laboratory of Design for Cognition, EDF R\&D in Clamart (France). In each office space, five members of a distributed team were equipped with pre-versions of the Personal.Aura artefact. All participants had personal symbols assigned to them that were shown on the remote Hello. Wall, each time they entered the local common area. The individual symbols were designed to overlay the ambient patterns, which continuously display the average mood and activity level of the team.

To prepare the ground for informal face-to-face communication, the test installation aimed at supporting the team members on both sides in approaching each other by successive signals of agreement, before actually engaging in a conversation. Therefore, special 'request buttons' were installed, which could be used to express the interest for a video communication with remote users. Pressing the request button 
results in an attention-catching pattern, which is shown on the Hello.Wall at the remote site. The overall mood of each team was captured with an easy, but very effective three-button interface. After one of the 'mood buttons' (bad, average or good) is pressed, its respective value is added to the overall mood of the local team, and the updated mood pattern appears on the Hello. Wall in the remote common area. Thus, the Hello.Wall continuously presents an intuitively perceivable picture about the atmosphere at the remote site in an ambient and unobtrusive way. In addition, webcams were installed at both sides to provide short glances into the remote common area. The webcams could be accessed from the remote side using a View.Port, which provide users with more-detailed information about the current situation in the remote lcommon area. To avoid misuse, a special pattern is displayed at the Hello.Wall, if a remote colleague is using a View.Port to glance into the common area.
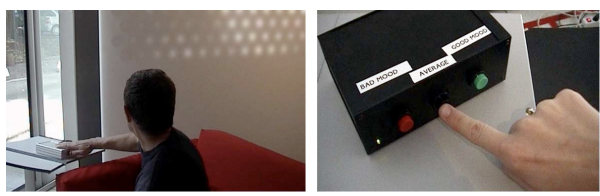

Fig. 4. Request button (left) and mood button (right)
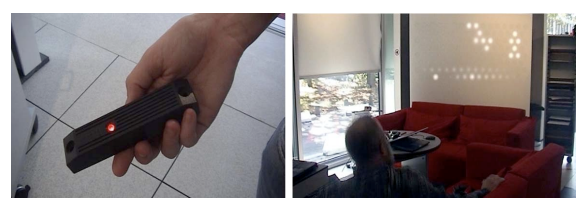

Fig. 5. Person detected via the Personal.Aura (left), and personal signs showing the presence of two colleagues in the remote common area (right)

The members of the distributed team were engaged in a joint activity of preparing a final report for a multi-national project. Additionally to this task, all participants were also collaborating with local colleagues, who were not part of the distributed team. All employees were using the same local common area, but only the members of the distributed team were equipped with Personal.Aura artefacts, and were familiar with the meaning of the team patterns. The participants were asked to press one of the mood buttons every time they come into the common area, and when entering or leaving the office building.

The observation and evaluation took place over a period of three weeks for three days a week. Each morning and afternoon the participants filled out a daily questionnaire, explaining their personal mood, and judging the perceived atmosphere and activity of the remote team. In an additional weekly questionnaire, which was given to the participants at the end of the week, they were asked more general questions about the usage of the Hello.Wall artefact, and their communication behavior with remote team members. After the evaluation period, all team members described their impressions and experiences in a final questionnaire, and evaluated the influence of the artefacts on the communication behavior of the team. In addition to the questionnaires, a camera system, mounted at the ceiling above the common area, was used to observe the behavior of the participants. Figure 6 shows some pictures taken by the observation cameras during the field study.

The results of the field test proved the effectiveness of the developed artefacts and confirmed its positive effects on workplace awareness and group communication. The 
data extracted from the questionnaires showed, that more interactions between both labs took place, and that the video communication system was used more often than before. The test installation was appreciated for providing a feeling for the atmosphere at the remote site and the number of people present, without disturbing the participants' privacy and workflow. User found it very helpful to see "who is there", and seemed to gain experience of how the remote colleagues work, and the way the lab is organized. The Hello.Wall was described as "a good measure to establish an everyday relationship with people, who are not physically present", and to improve the atmosphere in the lab "by taking it from isolation".

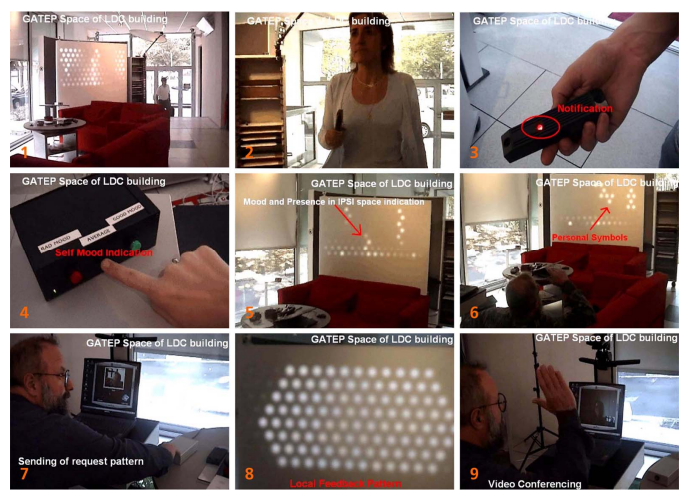

Fig. 6. Impressions of the field study: (1) common area at EDF, (2) user entering the common area, (3) identification via Personal.Aura prototype, (4) expression of current mood using the mood button interface, (5) Hello.Wall showing the overall mood and activity of the remote team members, (6) personal signs indicating the presence of two team members in the remote common area, (7) user sending communication request to remote colleagues, (8) local feedback pattern, (9) informal communication using the video-conferencing facilities

It could also be shown, that the Hello. Wall can serve as an unobtrusive awareness device in real-world working environments. While the members of the distributed team gained practical benefits using the Hello.Wall, the artefact did not attract any attention of people who were not participating in the joint activity, but eventually were spending some time in the common area around the Hello.Wall. Details of the evaluation can be found in [6].

\section{Conclusion}

The results of the evaluations led to the conclusion, that the predominant assumption of a fundamental trade-off in multi-user awareness systems is not tenable anymore. The evaluations verified the approach of this paper and showed that a cautious interface design can enhance user privacy in multi-user awareness systems and minimize disruptive effects on primary tasks, without reducing awareness mediation and usability. In addition, the developed artefacts successfully demonstrated that 
dedicated devices for capturing and representing awareness information in smart office environments have great potential to enhance the functionality as well as usability of multi-user awareness systems.

Acknowledgments. A number of people have contributed to the work described in this paper. We would like to thank all our current and former colleagues of the AMBIENTE research division at Fraunhofer IPSI. Especially Thorsten Prante, Daniel van Alphen and Daniela A. Plewe (for their inspiring 'Connecting-Remote-Teams' scenario), Richard Stenzel (for providing the software for the Hello.Wall driver interface and the various sample applications), as well as Dr. Dr. Norbert Streitz for his support. Special thanks are due to Daniel van Alphen for developing the Hello.Wall and View.Port artefacts, Sebastian Lex for the design of the Personal.Aura and Stefan Zink for his help on the Hello.Wall driver interface. We are especially grateful for the support we received from from Maral Memisoglu during the experimental evaluation as well as Dr. Saadi Lahlou, Dr. Valery Nosulenko, Dr. Lena Samoylenko during the field study.

\section{References}

1. Dourish, P., Bly, S.: Portholes: Supporting Awareness in a Distributed Work Group. In: Proceedings of the ACM Conference on Human Factors in Computing Systems (CHI'92), pp. 541-547 (1992)

2. Hudson, S.E., Smith, I.: Techniques for Addressing Fundamental Privacy and Disruption Tradeoffs in Awareness Support Systems. In: Proceedings of the ACM Conference on Computer Supported Cooperative Work (CSCW'96), pp. 248-257 (1996)

3. Kraut, R.E., Fish, R.S., Root, R.W., Chalfonte, B.L.: Informal Communication in Organizations: Form, Function, and Technology. In: Oskamp, S., Spacapan, S. (eds.) Human Reactions to Technology: The Claremont Symposium on Applied Social Psychology. Beverly Hills, CA, Sage Publications, Thousand Oaks, pp. 145-199. Reprinted in: Baecker, R.M. (ed.) Readings in Groupware and Computer-Supported Cooperative Work, Morgan Kaufmann, San Francisco, CA, pp. 287-314 (1990)

4. Memisoglu, M.: Between Awareness and Distraction - Comparison of an Abstract and a Concrete Information Representation of Presence Information as a Content Example for an Awareness Tool. Diploma Thesis, Technical University of Darmstadt, Germany.Dourish, P., Adler, A., Bellotti, V., Henderson, A. (eds.) (1996) Your Place or Mine: Learning from Long-Term Use of Audiovideo Communication. In: Computer Supported Cooperative Work, vol. 5(1), pp. 33-62 (2004)

5. Milewski, A., Smith, T.: Providing Presence Cues to Telephone Users. In: Proceedings of the ACM Conference on Computer Supported Cooperative Work (CSCW'00), pp. 89-96 (2000)

6. Nosulenko, V., Samoylenko, E., Welinski, P.: D12.10 - Hello.Wall and Videomaton User Experience. Deliverable of the 'Ambient Agoras' Project for the Third Disappearing Computer Jamboree, Ivrea, Italy (2003)

7. Olson, G.M., Olson, J.S.: Distance Matters. Human-Computer Interaction 15(2-3), 139178 (2000)

8. Olson, M.H., Bly, S.A.: The Portland Experience: A Report on a Distributed Research Group. In International Journal of Man.-Machine Studies 34(2), 211-228 (1991) 
9. Prante, T., Stenzel, R., Röcker, C., van Alphen, D., Streitz, N.A., Magerkurth, C., Plewe, D.A.: Connecting Remote Teams: Cross-Media Integration to Support Remote Informal Encounters. In: Davies, N., Mynatt, E.D., Siio, I. (eds.) UbiComp 2004. LNCS, vol. 3205, Springer, Heidelberg (2004)

10. Röcker, C.: Awareness and Informal Communication in Smart Office Environments. Verlag Dr. Driesen, Taunusstein, Germany. ISBN 3-936328-65-X (2006)

11. Röcker, C., Prante, T., Streitz, N.A., van Alphen, D.: Using Ambient Displays and Smart Artefacts to Support Community Interaction in Distributed Teams. In: Proceedings of the OZCHI Conference (OZCHI'04), University of Wollongong, Australia, ISBN 1-74128079, CD-ROM (November 22-24,2004)

12. Streitz, N.A., Prante, T., Röcker, C., van Alphen, D., Magerkurth, C., Stenzel, R., Plewe, D.A.: Ambient Displays and Mobile Devices for the Creation of Social Architectural Spaces: Supporting informal communication and social awareness in organizations. In: O’Hara, K., Perry, M., Churchill, E., Russell, D. (eds.) Public and Situated Displays: Social and Interactional Aspects of Shared Display Technologies, pp. 387-409. Kluwer Publishers, Dordrecht (2003a)

13. Tang, J.C., Isaacs, E.A., Rua, M.: Supporting Distributed Groups with a Montage of Lightweight Interactions. In: Proceedings of the ACM Conference on Computer Supported Cooperative Work (CSCW'94), pp. 23-34 (1994) 